\title{
Resolution-enhanced optical coherence tomography based on classical intensity interferometry
}

\author{
Hanna Lajunen, ${ }^{1}$ Víctor Torres-Company, ${ }^{2,3, *}$ Jesús Lancis, ${ }^{2}$ and Ari T. Friberg ${ }^{4,5,6}$ \\ ${ }^{1}$ Departamento de Óptica, Universidad de Valencia, ES-46100 Burjasot, Spain \\ ${ }^{2}$ Departament de Física, Universitat Jaume I, ES-12080 Castelló, Spain \\ ${ }^{3}$ Now at the Department of Electrical and Computer Engineering, McGill University, 3480 University Street, \\ H3A 2 A7 Montreal, Canada \\ ${ }^{4}$ Department of Applied Physics, Helsinki University of Technology, P.O. Box 3500, FI-02015 TKK, Finland \\ ${ }^{5}$ Department of Physics and Mathematics, University of Joensuu, P.O. Box 111, FI-80101 Joensuu, Finland \\ ${ }^{6}$ Also at the Department of Microelectronics and Applied Physics, Royal Institute of Technology (KTH), \\ Electrum 229, SE-164 40 Kista, Sweden \\ *Corresponding author: victor.torrescompany@mail.mcgill.ca
}

Received October 27, 2008; revised February 7, 2009; accepted February 8, 2009; posted February 19, 2009 (Doc. ID 103281); published March 31, 2009

\begin{abstract}
We propose a fourth-order interference scheme for optical coherence tomography operating with broadband incoherent (or quasi-incoherent) light. It is shown that using this proposal, an axial resolution improvement by a factor of $\sqrt{2}$ and a better sensitivity for weakly reflecting samples are obtained than with the standard second-order correlation scheme. From a practical perspective, we suggest the use of broadband Q-switched pulses and performing ultrafast intensity correlation with a nonlinear crystal. The global performance of our proposal is illustrated by means of numerical simulations. (c) 2009 Optical Society of America

OCIS codes: $030.1640,170.4500,120.3180$.
\end{abstract}

\section{INTRODUCTION}

Optical coherence tomography (OCT) is a high-resolution noncontact imaging technique for cross-sectional mapping of inhomogeneous samples [1]. Applications cover topics from imaging of biological tissue to materials research $[2,3]$. Enhancing the axial resolution while causing minimum photodamage in the sample is crucial in order to achieve a better performance. In the time-domain OCT (TD-OCT) setup [see Fig. 1(a)], the second-order coherence properties of the illumination source allow effective sectioning of a reflective sample with an axial resolution that is proportional to the coherence time of the source. Therefore within the conventional OCT, the most accurate results can be obtained by using sources with wide spectral widths $[4,5]$.

In the past few years, different attempts have been made to improve the axial resolution of OCT measurements. Quantum-optical coherence tomography (Q-OCT) makes use of an entangled twin-photon (nonclassical) light source to achieve an enhancement in resolution by a factor of 2. Additionally, it leads to cancellation of the harmful dispersion effects in the sample [6,7]. Due to the low power levels of current entangled photon sources, efforts have been made to simulate the advantages of Q-OCT using classical light sources. Similar features have been reported in so-called phase-conjugate optical coherence tomography (PC-OCT) using a double-pass setup with a broadband phase conjugator in one arm of the interferometer [8] or, alternatively, by detection and manipulation of intensity spectra [9].
As another way to improve the results of conventional OCT, second-harmonic interference fringe signals, excited using femtosecond laser pulses, have been demonstrated to yield an intermediate resolution enhancement by a factor of $\sqrt{2}$. The key mechanism of this second-harmonic optical coherence tomography (SH-OCT) is the quadratic power dependence of the second-harmonic signal on the refractive index, which leads to an interference signal occurring at exactly double the frequency of the fundamental interference [10]. In contrast to TD-OCT, information is obtained only from materials with suitable nonlinear optical response. On the other hand, optical ranging of biological samples is also possible by measuring the cross correlation of femtosecond pulses with a nonlinear crystal [11]. A similar nonlinear detection scheme with broadband temporally coherent illumination has also been recently proposed to improve OCT resolution and signal-tonoise ratio [12].

We note that the resolution improvements in the previous methods are due to the detection of the fourth-order correlations recorded electronically, optically, or by numerical means. It is the aim of this work to show that any fourth-order interferometer such as the one shown in Fig. 1(b) leads to an improved resolution by a factor of $\sqrt{2}$ in axial scanning when a broadband classical spectrally incoherent light source is employed. One may realize that this original configuration is just a modified version of the classical Hanbury Brown-Twiss (HBT) interferometer [13]. The key is that, by assuming that the classical incoherent light source obeys Gaussian statistics, the fourth- 


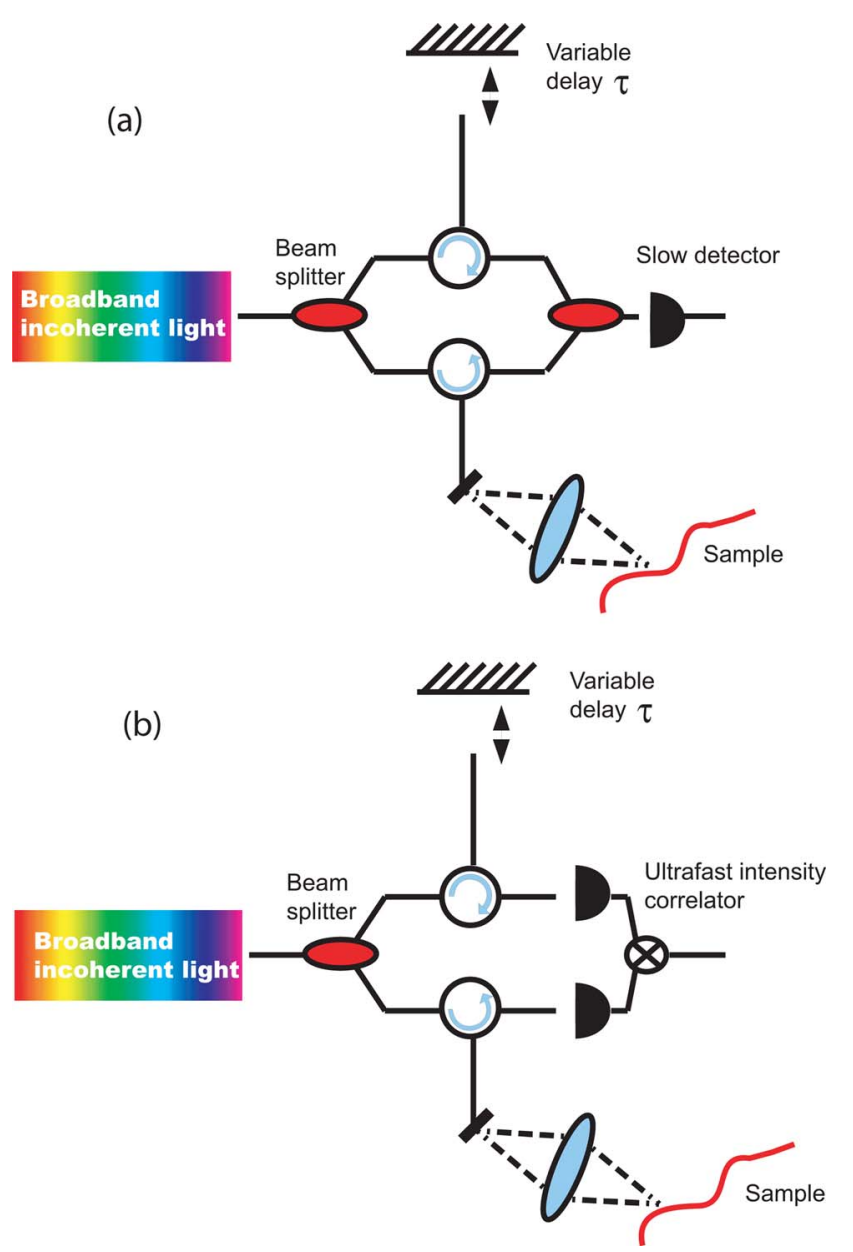

Fig. 1. (Color online) (a) Conventional time-domain OCT setup. (b) OCT device based on intensity interferometry with incoherent light.

order interference term can be written in terms of the interferogram that is achieved within the TD-OCT scheme shown in Fig. 1(a).

In Section 2, we study comparatively the expected signals in these two different schemes. We stress that the theoretical results are independent of the technique by which the intensity correlation is implemented. From a practical point of view, the resolving time of the intensity correlator in the modified HBT interferometer limits the coherence time of the stationary source. In fact, only sources with a coherence time much greater than the resolving time of the detecting scheme should be employed [14]. For common light sources employed in OCT, the coherence time is of the order of femtoseconds, which, from a practical point of view, requires the use of ultrafast detecting schemes. While our theory predicts the best results with stationary light, the limited response of the current electronics may require the use of high-power quasi-stationary light pulses as the ideal candidates to perform all-optical ultrafast intensity correlation. The main practical issues are discussed in Section 3. Numerical examples considering stationary and quasi-stationary light are illustrated in Section 4 and a comparative study with previous OCT techniques based on higher-order correlations is given in Section 5. Finally, in Section 6 we summarize the main goals of the work.

\section{THEORY}

For the sake of completeness, we recall first the basic equations of TD-OCT and compare them with our proposal. It will be shown that the intensity-correlation measurement provides a meaningful trace containing information on the layer structure similar to that obtained with the TD-OCT technique.

\section{A. Time-Domain Optical Coherence Tomography}

The conventional TD-OCT system is based on a secondorder (field) interferometer, where the reflecting sample with spectral transfer function $H(\omega)$ is set in one arm and a reference mirror in the other, as illustrated in Fig. 1(a). The interference between the reflected sample and reference fields is measured as a function of an adjustable time delay $\tau$ in the reference arm. We assume a stationary broadband source whose spectral density is denoted by $S\left(\omega+\omega_{0}\right)$, where $\omega_{0}$ is the central angular frequency. The measured interference signal then is of the form [2]

$$
I(\tau)=I_{s}+I_{r}+2 \Re\left\{\Gamma(\tau) \exp \left(-i \omega_{0} \tau\right)\right\},
$$

where

$$
I_{s}=\int S(\omega)\left|H\left(\omega+\omega_{0}\right)\right|^{2} \mathrm{~d} \omega
$$

and

$$
I_{r}=\int S(\omega) \mathrm{d} \omega
$$

are the constant background intensities of the sample and reference fields, respectively, and $\mathfrak{R}\{\}$ denotes the real part. Furthermore, $\Gamma(\tau)=\left\langle U_{s}^{*}(t) U_{r}(t+\tau)\right\rangle$, where the angle brackets denote time average, is the cross-correlation function between the complex fields $U_{s}$ and $U_{r}$. With the used notations, $\Gamma$ can be expressed as

$$
\Gamma(\tau)=\int H\left(\omega_{0}+\omega\right) S(\omega) \exp (-i \omega \tau) \mathrm{d} \omega,
$$

which contains the depth resolved structural data of the sample.

\section{B. Intensity-Correlation Optical Coherence Tomography with a Stationary Light Source}

In contrast to measuring field correlations as in conventional OCT, we propose replacing the second-order interferometer of the setup discussed above by a fourth-order (intensity) interferometer used for measuring the cross correlations of the intensities from each arm. Assuming that the statistics of the source field are Gaussian, higherorder correlation functions can be expressed in terms of second-order correlations [15]. Thus, the correlation of the intensity fluctuations is proportional to the squared modulus of the second-order coherence function, and a signal similar to that in conventional OCT can thus be expected. It is interesting to note the similarity of this setup to the original HBT interferometer [13]. HBT interferometers were introduced for measuring stellar diameters based on the intensity correlations of spatially incoherent 
sources [16], providing a better resolution than the alternative Michelson interferometer setup with cruder optical equipment [15].

The proposed intensity-correlation OCT (IC-OCT) setup is displayed in Fig. 1(b). The light from the source with spectrum $S\left(\omega+\omega_{0}\right)$ is split in a beam splitter into two paths consisting of the sample and an adjustable time delay, as before. Later, the cross correlation between these intensities is performed by either electronic or optical means and monitored as a function of the time delay. Assuming a classical stationary light source obeying Gaussian statistics, the intensity correlation function can be expressed as [15]

$$
C(\tau)=I_{s} I_{r}+|\Gamma(\tau)|^{2},
$$

where $I_{s}, I_{r}$, and $\Gamma(\tau)$ are now equal to the corresponding quantities defined for the TD-OCT case by Eqs. (2)-(4).

The main differences between these configurations can be found by comparing Eqs. (1) and (5). The TD-OCT signal is comparable to the real part of the second-order coherence function, while in the intensity-correlation measurement the squared absolute value of the same function is obtained. Thus the IC-OCT signal does not contain any information about the phase of $\Gamma$, but it can be used to determine the positions of the interfaces in layered samples with better accuracy than with normal OCT. It directly follows from the squared operation in IC-OCT that the resolution, defined as the inverse of the width of the signal envelope, is improved by a factor of $\sqrt{2}$. In addition, the constant background terms in Eqs. (1) and (5) are different, which will be seen to allow a better visibility in the intensity-correlation measurement, especially in the case of weakly reflecting samples.

\section{Intensity-Correlation Optical Coherence Tomography with a Quasi-Stationary Light Source}

Let us now consider the same fourth-order interferometer arrangement as in Fig. 1(b) but with a quasi-stationary light source [17] instead of a strictly stationary one. The need for this study will be clarified later. A quasistationary signal implies that the mutual coherence function no longer depends exactly on the time difference and that it is pulsed in time. Additionally, the intensity is a slowly varying function on a time scale of the order of the coherence time of the source, which is still provided by the inverse of the width of $S\left(\omega+\omega_{0}\right)$. Under these conditions and assuming that the source field statistics are Gaussian, it is easy to show that Eq. (5) should be replaced by

$$
C(\tau) \approx I_{s} I_{r} f(\tau)+|\Gamma(\tau)|^{2},
$$

where

$$
f(\tau)=\int \widetilde{I}(\omega) \widetilde{I}(-\omega) \exp (i \omega \tau) \mathrm{d} \omega
$$

and $\widetilde{I}(\omega)$ is the Fourier transform of the averaged intensity profile of the source. Equation (6) has the same structure as Eq. (5) apart from the fact that the background term now depends on the time delay. However, because of the quasi-stationarity properties, the $f(\tau)$ does not vary much in the time scale where $\Gamma(\tau)$ does, and therefore the influence (and structure) of these two different functions

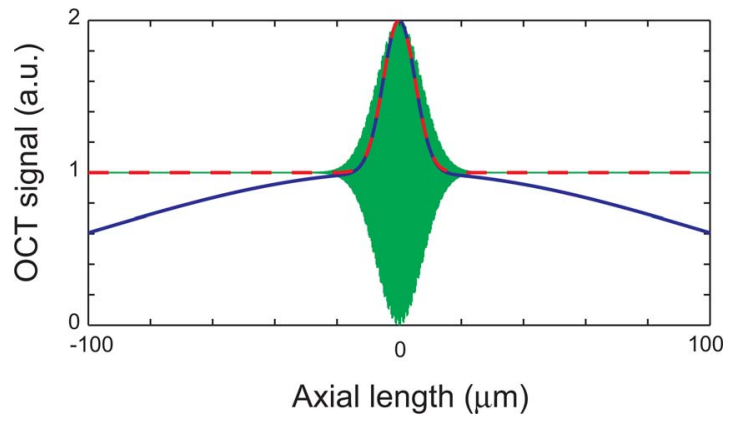

Fig. 2. (Color online) Simulation results for the normalized interferogram in TD-OCT (green solid curve) and the normalized IC-OCT using a stationary light source (red dashed curve) or quasi-stationary light source (blue thick solid curve). It corresponds to the devices of Fig. 1 for a single-layer sample. Normalization is taken with respect to the background of the corresponding interferogram. See details in text.

can be retrieved from the interferogram.

Let us clarify further the influence of the above three signals [Eqs. (4)-(6)] by illustrating their features with the simplest example of having a purely reflecting sample, assuming a Gaussian spectrum centered on $800 \mathrm{~nm}$ with $11 \mathrm{~nm}$ spectral width. This corresponds approximately to $11 \mathrm{fs}$ coherence time. For the quasistationary signal example, a Gaussian pulse with the same spectrum but with $1 \mathrm{ps}$ temporal width is considered. Note that the temporal intensity distribution of quasi-stationary signal does not affect the energy spectrum. Figure 2 displays the achieved results. As advanced, IC-OCT offers a $\sqrt{2}$ better resolution than conventional OCT. In the case of having quasi-stationary pulses, the relevant signal appears over a "pedestal," as predicted by Eq. (6). In other words, the narrow "coherence spike" contains the information about the coherence properties of the field, and therefore the useful information about the sample, whereas the pedestal contains the information about the temporal pulse duration [18]. For quasistationary pulses, where the coherence time is much shorter than the pulse duration, these two different contributions are clearly distinguishable. Thus, in practical terms, quasi-stationary light pulses can be used for IC-OCT as an alternative to strictly stationary light.

\section{PRACTICAL CONSIDERATIONS}

Here we consider some important practical aspects concerning the performance, light sources, and detectors that could match the previous theoretical requirements.

\section{A. Sources}

The theoretical results obtained for the intensitycorrelation signal were derived under the assumption of light source obeying Gaussian statistics, which allows us to express the higher-order intensity-correlation function in terms of the second-order correlations of the fields [15]. In practice, it is not easy to determine the statistical properties of optical sources. However, according to the central limit theorem, any random process that is a result of many additive and independent random events follows Gaussian statistics asymptotically [19]. Thus, it is reasonable to expect that our assumption is valid for many com- 
mon light sources used in conventional OCT, such as superluminescent diodes (SLDs) and other thermal sources [2]. Pulses from mode-locked lasers are, within a very good approximation, spectrally coherent and are not expected to obey Gaussian statistics.

For IC-OCT performed with quasi-stationary light pulses, convenient candidates would be Q-switching laser pulses. The inherent broadband nature and high peak power of these pulses would be ideal for performing nonlinear optical processes, allowing for an ultrafast detection scheme. It is important to note that because the broad spectrum of these pulses is not related to their relatively long temporal pulse duration $(\sim n s)$, this kind of source has been modeled as quasi-stationary and used for pump-probe spectroscopy as an alternative to coherent sources [20]. Additionally, recent advances in supercontinuum generated in photonic crystal fibers pumped with $\mathrm{Q}$-switched pulses suggest that they could trigger important applications in OCT [21] and even compete in terms of axial resolution with other coherent supercontinuum sources [5].

\section{B. Detection Process}

The intensity cross-correlation scheme from Fig. 1(b) ideally should be ultrafast. When implemented by electronic means, the finite bandwidth of the photodiode, which might approach $80 \mathrm{GHz}$, would be the ultimate bottleneck in tracking the random intensity variations that typically take place at the order of the coherence time of the source [15], limiting by several orders of magnitude the available optical bandwidth from superluminescent LEDs (S-LEDs) or Q-switched pulses and therefore degrading the axial resolution.

Ultrafast intensity correlation could be performed by all-optical means, using for example sum-frequency or second-harmonic generation (SHG). In SHG, assuming ideal phase-matching conditions, the upconverted optical field is proportional to the product of the sample and reference electric fields. By filtering the upconverted signal, detecting it with a slow response photodiode, and repeating the operation for different delays $\tau$, one would obtain the desired intensity-correlation trace given by Eqs. (5) and (6), depending on the type of source employed. Ideal broadband phase-matching conditions are achieved in thin nonlinear crystals at the expense of the efficiency of the process. Therefore, a high-power source would be required. For this case, Q-switched quasi-stationary pulses would offer a better performance with respect to continuous-wave low-power S-LEDs. SHG generated with incoherent sources has been previously reported [22,23], offering an efficiency twice as good as a coherent source with the same average power [23]. It is interesting to mention that parametric nonlinear processes can be excited, too, in thick nonlinear crystals [12], but in this case, a careful control of the relative spectral phase between the reference and sample pulses is required.

\section{Sensitivity}

One of the most important aspects to consider in OCT is the sensitivity to weakly reflecting samples [2]. We point out that IC-OCT is more sensitive in this respect than standard OCT due to the different background terms. In order to get further insight into this effect, let us consider a nondispersive sample consisting of a single surface with reflectance $r$. We now define the visibility as the maximum of the signal term divided by the constant background, i.e., $V_{\mathrm{TD}-\mathrm{OCT}}=\max [2|\Gamma(\tau)|] /\left(I_{r}+I_{s}\right)$ for the TD-OCT scheme and $V_{\text {IC-OCT }}=\max \left[|\Gamma(\tau)|^{2}\right] /\left(I_{r} I_{s}\right)$ for our implementation. Then in the conventional OCT case the visibility can be shown to take on value $2 r /\left(1+r^{2}\right)$. In contrast, the visibility of the IC-OCT signal in the case of single reflection has a constant value of 1 , independent of the layer reflectance. The importance of this property can easily be seen by noticing that for $r \rightarrow 0$ the normal OCT signal disappears while the normalized IC-OCT signal remains unaffected.

\section{Speed}

The time required to get a $3 \mathrm{D}$ image would be

$$
T=n_{x} n_{y} n_{z} T_{\tau}
$$

where $n_{j}$ is the number of pixels in dimension $j$ and $T_{\tau}$ the time necessary to record a signature at specific time $\tau$. Assuming that each Q-switch pulse contains enough energy to be detected and that the time integration is shorter than the period of the pulse train $T^{\prime}$, we get

$$
T_{\tau}=N T^{\prime},
$$

where $N$ is the number of pulses required to have a statistically significant sample to perform the average in the cross correlation. Taking $N=10^{3}, 1 \mathrm{MHz}$ repetition rate, and $128 \times 128$ pixels, we would require $16 \mathrm{~s}$ with our technique. Note that this time is the same for both the electronic and the all-optical detection schemes.

\section{NUMERICAL EXAMPLE}

To demonstrate the resolution improvement of the intensity-correlation measurement, we compare the expected OCT and IC-OCT signals of a sample structure based on numerical simulations. We consider layered, lossless dielectric samples whose spectral complex transfer function can be expressed as [3]

$$
H(\omega)=\sum_{n=0}^{N} r_{n} \exp \left[i 2 \sum_{k=1}^{n} \beta_{k}(\omega) L_{k}\right],
$$

where $r_{n}$ is the reflection coefficient and $L_{n}$ the thickness of each layer $n$, and $N$ is the total number of layers. Further, we assume that the dispersion coefficients $\beta_{n}(\omega)$ are expanded to second order in $\omega$, i.e., in each layer $\beta_{n}\left(\omega_{0}\right.$ $+\omega) \approx \beta_{0 n}+\beta_{1 n} \omega+\beta_{2 n} \omega^{2} / 2$, where $\beta_{1 n}$ is the inverse group velocity and $\beta_{2 n}$ is the group velocity dispersion (GVD). In other words, the transfer function describes the overall reflection from all the surfaces of the sample, taking into account the cumulative optical path to each surface.

As an illustrative example, we consider a sample consisting of two layers of different dispersive materials. The reflection coefficients at normal incidence from the front and back surfaces of the layer are chosen to be $r_{0}=r_{1}=r_{2}$ $=0.1$, the thicknesses are $L_{1}=80$ and $L_{2}=200 \mu \mathrm{m}$, and the corresponding GVD coefficients are $\beta_{21}=40$ $\times 10^{-24} \mathrm{~s}^{2} \mathrm{~m}^{-1}$ and $\beta_{22}=10 \times 10^{-24} \mathrm{~s}^{2} \mathrm{~m}^{-1}$. The values of $\beta_{0 i}$ and $\beta_{1 i}$ do not affect the normalized simulation re- 


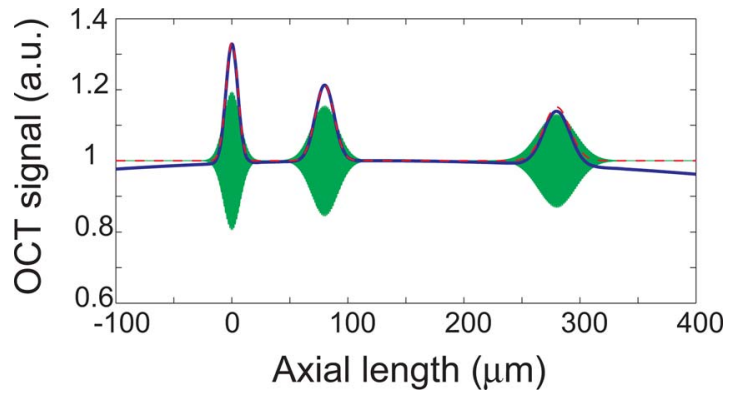

Fig. 3. (Color online) Simulation results for the normalized interferogram in TD-OCT (green solid curve) and the normalized IC-OCT using a stationary light source (red dashed curve) or quasi-stationary light source (blue thick solid curve). A two-layer dielectric sample is considered. See details in text.

sults. Note that the values of $\beta_{2 i}$ are actually $10^{3}$ times higher than the dispersion of fused silica, and thus the effects of GVD are strongly emphasized in the example.

The results of the simulation are illustrated in Fig. 3, where the conventional TD-OCT and IC-OCT signals (with the stationary and quasi-stationary case), normalized against their respective constant backgrounds, are plotted against the delay scaled by half the group velocity, $1 / 2 \beta_{1}$. The optical source for the TD-OCT and the IC-OCT working with stationary light is considered to be a S-LED with $11 \mathrm{~nm}$ bandwidth centered on $800 \mathrm{~nm}$ with Gaussian distribution. The thick solid curve (blue online) in Fig. 3 plots the IC-OCT trace for a Q-switch Gaussian pulse of $10 \mathrm{ps}$ temporal width and spectrum identical to that of the S-LED. The traditional OCT signal shows three peaks that correspond to reflections from the surfaces of the sample. The second and third peaks are stretched due to dispersion within the sample. The intensity-correlation signal exhibits corresponding peaks in the same positions, without the rapid phase variations in the signal. The resolution enhancement of the IC-OCT method is a factor of $\sqrt{2}$, as predicted. It is interesting to see the overlapping of the pedestals in the IC-OCT with the quasi-stationary Q-switch pulses. Despite this overlapping, it is the coherence spike of the intensity correlation at each reflection that contains the useful information about the samples. In fact, we note that the stationary and quasi-stationary cases in the IC-OCT are almost indistinguishable. It is also worth noticing that in the considered case of relatively weakly reflecting sample, the visibility of the normalized IC-OCT signal is better than that of the conventional OCT due to the different background terms. In fact, this would be seen even more clearly if a smaller reflection coefficient were chosen for all the layers.

\section{DISCUSSION}

Finally, let us compare our proposed intensity-correlation OCT with some of the other possible resolutionimprovement schemes mentioned in the Introduction. As a first notion we remind that the most common way to improve resolution in OCT measurements is to increase the bandwidth of the source. The same opportunity applies naturally also to the intensity-correlation measurements, while the relative improvement by a factor of $\sqrt{2}$ com- pared with the conventional OCT performed using the same source always remains.

The Q-OCT provides a resolution improvement by a factor of 2, and additionally the dispersion effects caused by the light propagation in the sample are canceled. However, to achieve these advantages, an entangled twophoton source is required, which limits the practical realizability of the method in view of the low photon flux of current quantum light sources. We emphasize that even though the dispersion is not completely canceled out in the intensity-correlation signal, the relative resolution improvement remains the same also in dispersive media. Moreover, numerical methods similar to those used in conventional OCT [24-26] could be developed to compensate for the dispersion effects also in the case of the intensity-correlation signals.

Since we have recently shown that some of the quantum phenomena based on the temporal entanglement of two-photon pairs can be emulated using classical thermal sources [27], one is intrigued by whether the advantages of Q-OCT could be achieved in the same way with classical light. However, even though nonlocal dispersion cancellation indeed is possible also with classical light in some cases [28], it turns out not to apply to the Hong-OuMandel (HOM) interferometer [29] setup used in Q-OCT. Thus arrangements more complicated than simply changing the source must be used in order to obtain the classical dispersion cancellation in OCT [8,9]. Nevertheless, using a classical thermal source in the corresponding HOM setup does give a signal that is reminiscent of the one obtained in Q-OCT, with resolution improved by a factor of $\sqrt{2}$. Differing from the HBT interferometer proposed here, the classical HOM-OCT signal contains oscillations at twice the frequency of the conventional OCT signal, and it is affected by additional interference terms between the actual reflection peaks similar to those obtained with the Q-OCT signals.

Compared with the SH-OCT [10], which also gives resolution improvement by a factor of $\sqrt{2}$, our proposal has some advantages. Namely, while IC-OCT can be realized using incoherent broadband sources similar those used with normal OCT, in SH-OCT a coherent high-power source is needed to excite the second-harmonic signal in the sample. This restricts the technique to use only for samples that are able to emit $\mathrm{SH}$ radiation, which is not straightforward in practice. In addition, the high power required to excite this nonlinear phenomenon might damage the sample. However, in our technique the nonlinear detection scheme is implemented not in the sample but in a nonlinear crystal with suitable characteristics. On the other hand, in the case of high-power quasi-stationary pulses, the intensity correlation signal could also be detected using nonlinear schemes similar to those demonstrated previously [11] with coherent pulses. Such an alloptical detection alternative would improve on the limitations set by digital processing.

\section{SUMMARY}

We have introduced a new noninvasive optical coherence tomography technique based on ultrafast intensity correlation between the sample and reference signals. With re- 
spect to previous approaches using broadband coherent or quantum sources, we have shown that our technique can be used with broadband spectrally incoherent (stationary) or quasi-incoherent (quasi-stationary) light pulses without any significant difference. Since ultrafast correlation is required, the electronic bandwidth of current photodiodes prevents their use for high-resolution depth imaging. When optical intensity correlation is performed, highpower Q-switched pulses (quasi-stationary pulses) might be better candidates than S-LEDs (stationary light) due to the high-peak power of the former pulses. This proposed setup enhances the resolution of conventional OCT by a factor of $\sqrt{2}$ and increases the sensitivity when the sample is composed of weakly reflective layers.

\section{ACKNOWLEDGMENTS}

The authors are grateful to J. P. Torres for bringing to their attention $[22,23]$. This work was partially funded by the Ministerio de Educacion y Ciencia (MEC), Spain, through grants TEC2005-07336-C02-02/MIC, FIS200762217, and CONSOLIDER CSD-2007-00013. V. T. thanks MEC for a Formación Profesorado Universitario grant. H. L. acknowledges support from the Juan de la Cierva program of MEC, Spain, and the Academy of Finland. A. T. F. received funding from the Swedish Foundation for Strategic Research (SSF) and the Academy of Finland under the Finland Distinguished Professor program.

\section{REFERENCES}

1. D. Huang, E. A. Swanson, C. P. Lin, J. S. Schuman, W. G. Stinton, W. Chang, M. R. Hee, T. Flotte, K. Gregory, C. A. Puliafito, and J. G. Fujimoto, "Optical coherence tomography," Science 254, 1178-1181 (1991).

2. A. F. Fercher, W. Drexler, C. K. Hitzenberger, and T. Lasser, "Optical coherence tomography-principles and applications," Rep. Prog. Phys. 66, 239-303 (2003).

3. P. H. Tomlins and R. K. Wang, "Theory, developments and applications of optical coherence tomography," J. Phys. D 38, 2519-2535 (2005).

4. W. Drexler, U. Morgner, F. X. Kartner, C. Pitris, S. A. Boppart, X. D. Li, E. P. Ippen, and J. G. Fujimoto, "In vivo ultrahigh-resolution optical coherence tomography," Opt. Lett. 24, 1221-1223 (1999).

5. I. Hartl, X. D. Li, C. Chudoba, R. K. Ghanta, T. H. Ko, J. G. Fujimoto, J. K. Ranka, and R. S. Windeler, "Ultrahighresolution optical coherence tomography using continuum generation in an air-silica microstructure optical fiber," Opt. Lett. 26, 608-610 (2001).

6. A. F. Abouraddy, M. B. Nasr, B. E. A. Saleh, A. V. Sergienko, and M. C. Teich, "Quantum-optical coherence tomography with dispersion cancellation," Phys. Rev. A 65, 053817 (2002).

7. M. B. Nasr, B. E. A. Saleh, A. V. Sergienko, and M. C. Teich, "Demonstration of dispersion-canceled quantumoptical coherence tomography," Phys. Rev. Lett. 91, 083601 (2003).
8. B. I. Erkmen and J. H. Shapiro, "Phase-conjugate optical coherence tomography," Phys. Rev. A 74, 041601 (2006).

9. K. J. Resch, P. Puvanathasan, J. S. Lundeen, M. V. Mitchell, and K. Bizheva, "Classical dispersion-cancellation interferometry,” Opt. Express 15, 8797-8804 (2007).

10. Y. Jiang, I. Tomov, Y. Wang, and Z. Chen, "Secondharmonic optical coherence tomography," Opt. Lett. 29, 1090-1092 (2003).

11. J. G. Fujimoto, S. De Silvestri, E. P. Ippen, C. A. Pulifiato, R. Margolis, and A. Oseroff, "Femtosecond optical ranging in biological systems," Opt. Lett. 11, 150-152 (1986).

12. A. Pe'er, Y. Bromberg, B. Dayan, Y. Silberberg, and A. A. Friesem, "Broadband sum-frequency generation as an efficient two-photon detector for optical tomography," Opt. Express 15, 8760-8769 (2007).

13. R. Hanbury Brown and R. Q. Twiss, "Correlation between photons in two coherent beams of light," Nature 177, 27-32 (1956).

14. Z. Y. Ou, E. C. Gage, B. E. Magill, and L. Mandel, "Fourthorder interference technique for determining the coherence time of a light beam," J. Opt. Soc. Am. B 6, 100-103 (1989).

15. L. Mandel and E. Wolf, Optical Coherence and Quantum Optics (Cambridge U. Press, 1995).

16. R. Hanbury Brown and R. Q. Twiss, "A test of a new type of stellar interferometer on Sirius," Nature 178, 1046-1048 (1956).

17. R. A. Silverman, "Locally stationary random processes," IRE Trans. Inf. Theory 3, 182-187 (1957).

18. J. C. Diels and W. Rudolph, Ultrashort Laser Pulse Phenomena (Academic, 1996).

19. J. W. Goodman, Statistical Optics (Wiley-Interscience, 1985).

20. M. Tomita and M. Matsuoka, "Ultrafast pump-probe measurement using intensity correlation of incoherent light," J. Opt. Soc. Am. B 3, 560-563 (1986).

21. W. Wadsworth, N. Joly, J. Knight, T. Birks, F. Biancala, and P. Russell, "Supercontinuum and four-wave mixing with Q-switched pulses in endlessly single-mode photonic crystal fibers," Opt. Express 12, 299-309 (2004).

22. M. C. Teich, R. L. Abrams, and W. B. Gandrud, "Photoncorrelation enhancement of SHG at $10.6 \mu \mathrm{m}$," Opt. Commun. 2, 206-208 (1970).

23. Y. Qu and S. Singh, "Photon correlation effects in second harmonic generation," Opt. Commun. 90, 111-114 (1992).

24. A. F. Fercher, C. K. Hitzenberger, M. Sticker, R. Zawadzki, B. Karamata, and T. Lasser, "Numerical dispersion compensation for partial coherence interferometry and optical coherence tomography," Opt. Express 9, 610-615 (2001).

25. D. L. Marks, A. L. Oldenburg, J. J. Reynolds, and S. A. Boppart, "Autofocus algorithm for dispersion correction in optical coherence tomography," Appl. Opt. 42, 3038-3046 (2003).

26. K. Banaszek, A. S. Randunsky, and I. A. Walmsley, "Blind dispersion compensation for optical coherence tomography," Opt. Commun. 269, 152-155 (2007).

27. V. Torres-Company, H. Lajunen, J. Lancis, and A. T. Friberg, "Ghost interference with classical partially coherent light pulses," Phys. Rev. A 77, 043811 (2008).

28. V. Torres-Company, H. Lajunen, and A. T. Friberg, "Nonlocal dispersion cancellation with classical light and its application to remote spectral transfer," (submitted to New J. Phys., 2009).

29. C. K. Hong, Z. Y. Ou, and L. Mandel, "Measurement of subpicosecond time intervals between two photons by interference," Phys. Rev. Lett. 59, 2044 (1987). 